\title{
Correction: Etelcalcetide in Patients on Hemodialysis with Severe Secondary Hyperparathyroidism. Multicenter Study in "Real Life". J. Clin. Med. 2019, 8,1066
}

\author{
Domenico Russo ${ }^{1, *}$, Rocco Tripepi ${ }^{2}$, Fabio Malberti ${ }^{3}$, Biagio Di Iorio ${ }^{4}$, \\ Bernadette Scognamiglio ${ }^{1}$, Luca Di Lullo ${ }^{5}$, Immacolata Gaia Paduano ${ }^{1}$, Giovanni Luigi Tripepi ${ }^{2}$ \\ and Vincenzo Antonio Panuccio ${ }^{6}$ \\ 1 Department of Public Health, University of Naples FEDERICO II, 80131 Naples, Italy; \\ bernadette.scognamig@alice.it (B.S.); gipaduano@libero.it (I.G.P.) \\ 2 Institute of Clinical Physiology (IFC-CNR) Research Unit of Reggio Calabria, 89124 Reggio Calabria, Italy; \\ rocco.tripepi@ifc.cnr.it (R.T.); gtripepi@ifc.cnr.it (G.L.T.) \\ 3 Department of Nephrology Cremona Hospital, 26100 Cremona, Italy; f.malberti@ospedale.cremona.it \\ 4 Department of Nephrology AORN Cardarelli, 80131 Naples, Italy; brdiiorio@gmail.com \\ 5 Department of Nephrology Ospedale “Parodi Delfino" di Colleferro (Roma), Colleferro, 00034 Roma, Italy; \\ dilulloluca69@gmail.com \\ 6 Nephrology, Dialysis and transplantation Unit G.O.M. "Bianchi Melacrino Morelli", \\ 89121 Reggio Calabria, Italy; enzopanuccio@gmail.com \\ * Correspondence: domenicorusso51@hotmail.com; Tel.: +39-335-738-4009
}

The authors wish to make the following corrections to the previous publication [1] in the text, Tables 1 and 2, and also Figure 1.

In the text on page 2 , it is reported that "The following levels of serum calcium were used for the definition of hypocalcemia: $<7.0 \mathrm{mEq} / \mathrm{L} ; \geq 7.0 \mathrm{but} \leq 7.5 \mathrm{mEq} / \mathrm{L} ; \geq 7.5 \mathrm{but}<8.3 \mathrm{mEq} / \mathrm{L}$ ".

This statement needs to be corrected: "The following levels of serum calcium were used for the definition of hypocalcemia: $<7.0 \mathrm{mg} / \mathrm{dL} ; \geq 7.0$ but $\leq 7.5 \mathrm{mg} / \mathrm{dL} ;>7.5$ but $<8.3 \mathrm{mg} / \mathrm{dL}$ ".

We wish to correct the caption of Table 1 where serum calcium concentrations are reported as $\mathrm{mEq} / \mathrm{L}$ instead of $\mathrm{mg} / \mathrm{dL}$. 
Table 1. Patients' Characteristics.

\begin{tabular}{|c|c|c|c|c|}
\hline & $\begin{array}{c}\text { Total Group } \\
(n=168)\end{array}$ & $\begin{array}{l}\text { Naïve Group } \\
\quad(n=56)\end{array}$ & $\begin{array}{l}\text { Switch Group } \\
\quad(n=112)\end{array}$ & $\begin{array}{c}p \\
\text { (Naïve vs Switch) }\end{array}$ \\
\hline Age (years) & $61 \pm 14$ & $64 \pm 14$ & $59 \pm 14$ & 0.04 \\
\hline Male (\%) & 57 & 52 & 60 & 0.32 \\
\hline Dialysis vintage (month) & 58 (IQR 32-102) & 35 (IQR 14-63) & 69 (IQR 48-120) & $<0.001$ \\
\hline Diabetes (\%) & 25 & 31 & 22 & 0.23 \\
\hline Cardiovascular comorbidities (\%) & 73 & 70 & 75 & 0.53 \\
\hline iPTH (pg/mL) & 636 (IQR 493-916) & 602 (IQR 509-800) & 664 (IQR 495-947) & 0.67 \\
\hline Serum Calcium $(\mathrm{mEq} / \mathrm{L})$ & $9.0 \pm 1.0$ & $9.1 \pm 0.7$ & $9.0 \pm 1.1$ & 0.60 \\
\hline Serum Phosphate (mg/dL) & $5.6 \pm 1.4$ & $5.5 \pm 1.4$ & $5.6 \pm 1.4$ & 0.83 \\
\hline Alkaline Phosphate (U.I./L) & 131 (IQR 83-201) & 111 (IQR 74-159) & 148 (IQR 88-221) & 0.02 \\
\hline $\mathrm{Hb}(\mathrm{gr} / \mathrm{dL})$ & $11.1 \pm 1.4$ & $11.0 \pm 1.2$ & $11.1 \pm 1.4$ & 0.58 \\
\hline ESA treatment $(\%)$ & 87 & 88 & 87 & 0.97 \\
\hline Phosphate binders therapy (\%) & 96 & 93 & 97 & 0.17 \\
\hline Calcium containing binders (\%) & 18 & 25 & 14 & 0.09 \\
\hline Vitamin D therapy (\%) & 75 & 83 & 71 & 0.09 \\
\hline Native Vitamin D therapy (\%) & 5 & 4 & 6 & 0.59 \\
\hline Previous cinacalcet treatment (\%) & 67 & 0 & 100 & N/A \\
\hline
\end{tabular}

IQR, interquartile range; iPTH, intact parathyroid hormone; $\mathrm{Hb}$, hemoglobin; ESA, erythropoietin-stimulating agent.

The caption of the amended Table 1 is:

Table 1. Patients' Characteristics.

\begin{tabular}{|c|c|c|c|c|}
\hline & $\begin{array}{l}\text { Total Group } \\
(n=168)\end{array}$ & $\begin{array}{l}\text { Naïve Group } \\
\quad(n=56)\end{array}$ & $\begin{array}{l}\text { Switch Group } \\
\quad(n=112)\end{array}$ & $\begin{array}{c}p \\
\text { (Naïve vs Switch) }\end{array}$ \\
\hline Age (years) & $61 \pm 14$ & $64 \pm 14$ & $59 \pm 14$ & 0.04 \\
\hline Male (\%) & 57 & 52 & 60 & 0.32 \\
\hline Dialysis vintage (month) & 58 (IQR 32-102) & 35 (IQR 14-63) & 69 (IQR 48-120) & $<0.001$ \\
\hline Diabetes (\%) & 25 & 31 & 22 & 0.23 \\
\hline Cardiovascular comorbidities (\%) & 73 & 70 & 75 & 0.53 \\
\hline iPTH (pg/mL) & 636 (IQR 493-916) & 602 (IQR 509-800) & 664 (IQR 495-947) & 0.67 \\
\hline Serum Calcium (mg/dL) & $9.0 \pm 1.0$ & $9.1 \pm 0.7$ & $9.0 \pm 1.1$ & 0.60 \\
\hline Serum Phosphate (mg/dL) & $5.6 \pm 1.4$ & $5.5 \pm 1.4$ & $5.6 \pm 1.4$ & 0.83 \\
\hline Alkaline Phosphate (U.I./L) & 131 (IQR 83-201) & 111 (IQR 74-159) & 148 (IQR 88-221) & 0.02 \\
\hline $\mathrm{Hb}(\mathrm{gr} / \mathrm{dL})$ & $11.1 \pm 1.4$ & $11.0 \pm 1.2$ & $11.1 \pm 1.4$ & 0.58 \\
\hline ESA treatment $(\%)$ & 87 & 88 & 87 & 0.97 \\
\hline Phosphate binders therapy (\%) & 96 & 93 & 97 & 0.17 \\
\hline Calcium containing binders ( $\%)$ & 18 & 25 & 14 & 0.09 \\
\hline Vitamin D therapy (\%) & 75 & 83 & 71 & 0.09 \\
\hline Native Vitamin D therapy (\%) & 5 & 4 & 6 & 0.59 \\
\hline Previous cinacalcet treatment (\%) & 67 & 0 & 100 & N/A \\
\hline
\end{tabular}

$\mathrm{IQR}$, interquartile range; iPTH, intact parathyroid hormone; $\mathrm{Hb}$, hemoglobin; ESA, erythropoietin-stimulating agent.

We wish to correct the caption of Table 2 where serum calcium concentrations are reported as $\mathrm{mEq} / \mathrm{L}$ instead of $\mathrm{mg} / \mathrm{dL}$ and etelcalcetide is reported as Parsabiv (trade name). 
Table 2. Cases of hypocalcemia.

\begin{tabular}{cccc}
\hline Days after Parsabiv & $<7.0 \mathrm{mEq} / \mathrm{L}$ & $\geq 7.0$ or $<7.5 \mathrm{mEq} / \mathrm{L}$ & $\geq 7.5$ or $<8.3 \mathrm{mEq} / \mathrm{L}$ \\
\hline 30 & $3 / 168(1.8 \%)$ & 0 & $25 / 168(14.9 \%))$ \\
\hline 60 & $1 / 129(0.8 \%)$ & $7 / 129(5.4 \%)$ & $28 / 129(21.7 \%)$ \\
\hline 90 & $2 / 111(1.8 \%)$ & $2 / 111(1.8 \%)$ & $27 / 111(24.3 \%)$ \\
\hline 120 & $1 / 80(1.3 \%)$ & $1 / 80(1.3 \%)$ & $21 / 80(26.2 \%)$ \\
\hline 150 & $1 / 61(1.6 \%)$ & $6 / 61(9.8 \%)$ & $11 / 61(18.0 \%)$ \\
\hline 180 & 0 & $1 / 44(2.3 \%)$ & $11 / 44(25.0 \%)$ \\
\hline 210 & 0 & $1 / 51(2.0 \%)$ & $15 / 51(29.4 \%)$ \\
\hline
\end{tabular}

The caption of the amended Table 2 is:

Table 2. Cases of hypocalcemia.

\begin{tabular}{cccc}
\hline Days after Etelcalcetide & $<7.0 \mathrm{mg} / \mathrm{dL}$ & $\geq 7.0$ or $\leq 7.5 \mathrm{mg} / \mathrm{dL}$ & $>7.5$ or $<8.3 \mathrm{mg} / \mathrm{dL}$ \\
\hline 30 & $3 / 168(1.8 \%)$ & 0 & $25 / 168(14.9 \%))$ \\
\hline 60 & $1 / 129(0.8 \%)$ & $7 / 129(5.4 \%)$ & $28 / 129(21.7 \%)$ \\
\hline 90 & $2 / 111(1.8 \%)$ & $2 / 111(1.8 \%)$ & $27 / 111(24.3 \%)$ \\
\hline 120 & $1 / 80(1.3 \%)$ & $1 / 80(1.3 \%)$ & $21 / 80(26.2 \%)$ \\
\hline 150 & $1 / 61(1.6 \%)$ & $6 / 61(9.8 \%)$ & $11 / 61(18.0 \%)$ \\
\hline 180 & 0 & $1 / 44(2.3 \%)$ & $11 / 44(25.0 \%)$ \\
\hline 210 & 0 & $1 / 51(2.0 \%)$ & $15 / 51(29.4 \%)$ \\
\hline
\end{tabular}

We wish to correct the caption of Figure 1 where serum calcium concentrations are reported as $\mathrm{mEq} / \mathrm{L}$ instead of $\mathrm{mg} / \mathrm{dL}$.
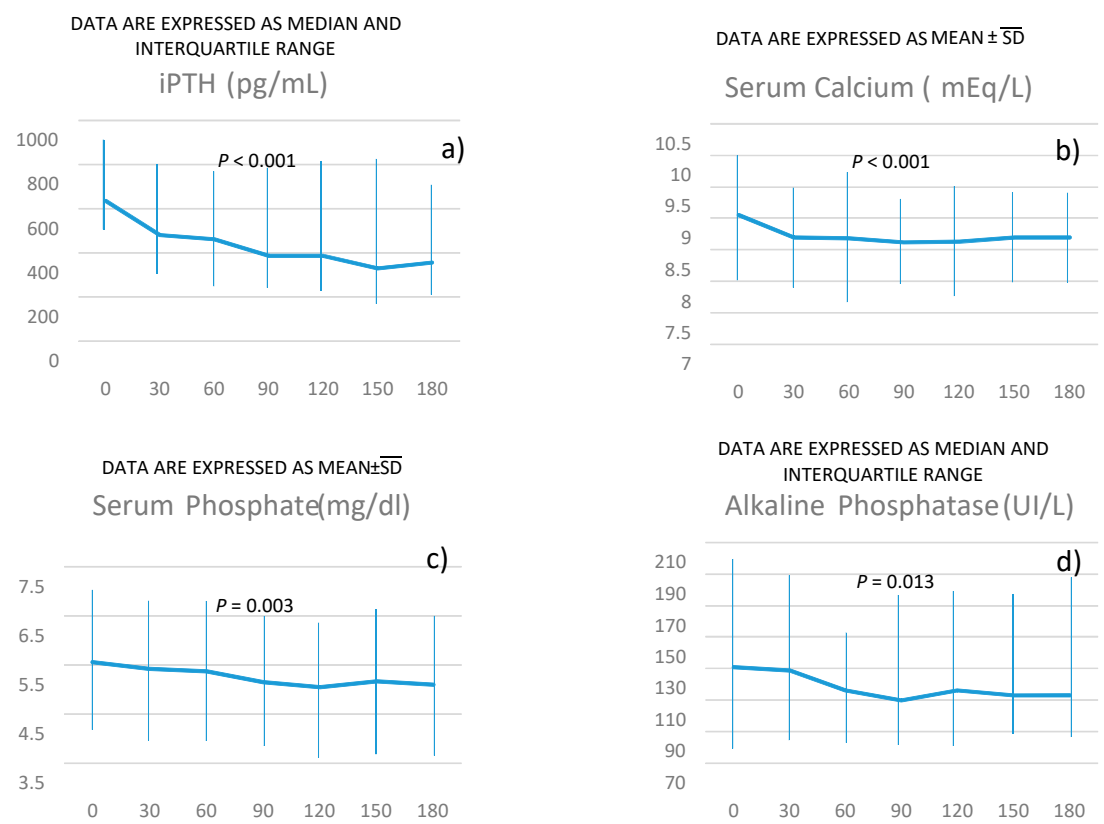

Figure 1. $P$ for the trend of intact parathyroid hormone (iPTH) (panel a), serum calcium (panel b), serum phosphate (panel c), alkaline phosphatase (panel d) over time was obtained using linear regression models weighted for patients' identification (see methods for more details). 
The caption of the amended Figure 1 is:

DATA ARE EXPRESSED AS MEDIAN AND INTERQUARTILE RANGE iPTH $(\mathrm{pg} / \mathrm{mL})$

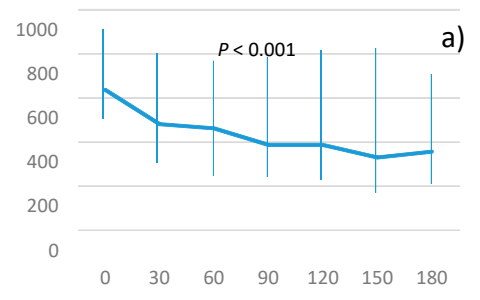

DATA ARE EXPRESSED AS MEAN $\pm \overline{S D}$ Serum Phosphate (mg/dL)

c)

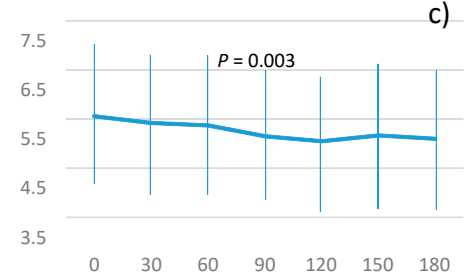

DATA ARE EXPRESSED AS MEAN $\pm \overline{\text { SD }}$

Serum Calcium (mg/dL)

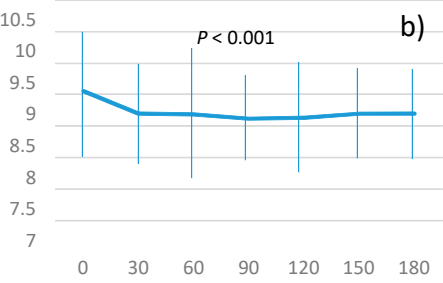

DATA ARE EXPRESSED AS MEDIAN AND INTERQUARTILE RANGE

Alkaline Phosphatase(UI/L)

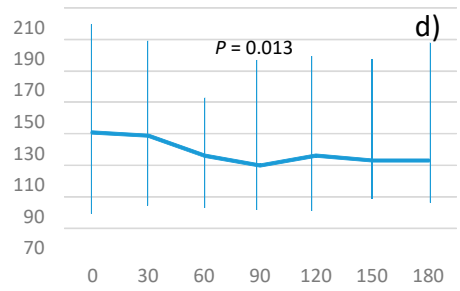

Figure 1. $P$ for the trend of intact parathyroid hormone (iPTH) (panel a), serum calcium (panel b), serum phosphate (panel c), alkaline phosphatase (panel d) over time was obtained using linear regression models weighted for patients' identification (see methods for more details).

The authors apologize to the readers for any inconvenience caused by these changes. It is important to state that this correction does not affect our study's results and involves no changes or modifications in the original data supporting our results. The original manuscript [1] will remain online on the article webpage, with reference to this Correction.

Conflicts of Interest: The authors declare no conflict of interest.

\section{Reference}

1. Russo, D.; Tripepi, R.; Malberti, F.; di Iorio, B.; Scognamiglio, B.; di Lullo, L.; Paduano, I.G.; Tripepi, G.L.; Panuccio, V.A. Etelcalcetide in Patients on Hemodialysis with Severe Secondary Hyperparathyroidism. Multicenter Study in "Real Life". J. Clin. Med. 2019, 8, 1066. [CrossRef] [PubMed] 